\title{
EDITORIAL
}

\section{Too indulgent or not sensitive enough: mothering in the current historical era and its relevance to childhood obesity}

\author{
International Journal of Obesity (2017) 41, 1457-1458; \\ doi:10.1038/ijo.2017.117
}

In this issue of the International Journal of Obesity, Anderson and colleagues ${ }^{1}$ describe results of their analysis testing a popular conceptual model for the prevention of childhood obesity: more structured home routines will lead to better self-regulation, which will prevent childhood obesity. There is a strong theoretical foundation for this model. Obesity-related hormones are entrained by daily routines, ${ }^{2}$ basic behavior theory suggests that routines increase child compliance ${ }^{3}$ and poor self-regulation has been repeatedly linked to childhood obesity. Unfortunately, the authors found that while some household routines were associated with some aspects of children's self-regulation, and some routines were associated with lower obesity risk, the child's self-regulation was not the mechanism of association. We likewise found that although a behavioral intervention improved children's self-regulation, these improvements did not reduce childhood obesity. ${ }^{4}$ A plethora of interventions seeking to improve parenting to prevent childhood obesity have now reported modest effects. ${ }^{5}$ Why might parenting interventions to prevent childhood obesity not be as effective as expected?

The lay press abounds with advice to parents to promote routines but the research evidence that doing so improves child outcomes is remarkably sparse. ${ }^{3}$ A potential explanation for modest effects of parenting interventions on child obesity may be that child characteristics moderate the effects of parenting on child outcomes in a transactional manner. For example, households with more routines have children with easier temperaments. ${ }^{6,7}$ These associations may be due to shared genetics between parents and child (both of whom may have rhythmic temperaments that easily adapt to routines) as opposed to parenting that causes children to have easier temperaments. Parenting routines are also more strongly associated with children's outcomes when the child is at biobehavioral risk. ${ }^{7,8}$ These observations align with the fact that most research on the effects of routines on children's outcomes has been among children at risk. ${ }^{9}$ The intervention potential of routines for typically developing, healthy children may be modest.

The current framing of parenting as a contributor to childhood obesity may also be a function of the era in history. The first half of the twentieth century saw the emergence of the "parenting expert' in the form of pediatricians or scientists publishing treatises on how women should most competently raise children. During this era, great emphasis was placed on structure, obedience and discipline. In the 1950's, there was an increased emphasis on sensitivity, responsiveness and attachment. The advent of the childhood obesity epidemic began introducing uncertainty about whether permissiveness, indulgence or lack of sensitivity could cause childhood obesity. ${ }^{10}$ There has therefore been increasing emphasis on teaching mothers to accurately read children's hunger and satiety cues based on the premise that if mothers accurately identified children's needs in a sensitive and responsive manner, children would not become obese. In direct contrast to this conceptual model, however, is emerging work that children's hunger and satiety cues cannot be accurately and reliably identified (by mothers or researchers). While early parenting culture emphasized rules, discipline and structure, and mid-twentieth century parenting emphasized sensitivity and attachment, the current parenting culture sends mixed messages rooted in the tension between permissiveness and discipline. ${ }^{11}$

A tension has also emerged related to the changing roles of women in society since the 1950's. In prior eras in history, fathers have typically been the strict disciplinarians imposing rules and structure while mothers have provided warmth, sensitivity and a strong sense of attachment security. In more recent years, fathers have been encouraged to take on a more significant role in being sensitive, responsive and nurturing parents. However, these cultural shifts have been more complicated for women. As women have entered the workplace, the pressure for women to become even more sensitive and nurturing at home has only increased. The stereotype of the working mother is one who is harried, rushed and has little time for sensitive nurturing of her children. Thus, there is conflicting societal messaging to women. On the one hand, they should focus on being sensitive and responsive to their child's needs; on the other hand, society gives the message that children are out of control and overindulged, and that there is a lack of structure, routine, discipline and expectations for self-control.

In the current culture of parenting, experts are uncomfortable suggesting directly that parents be 'stricter' and 'say no' more often (particularly with regard to denying children food). Instead of telling parents to be disciplinarians who must sometimes deny a child's wants, experts tell parents that if they help children to recognize hunger and satiety, and manage their emotions so they won't eat due to stress or provide children with a calm, nurturing, structured environment so that the children will fall into a predictable routine of eating and sleeping that children will not be obese. It is possible that this approach will have some effect. However, for the child at biological risk for obesity, who may simply be hungrier and less easily sated than the thinner child, or for the child whose physiology simply does not follow regular biological rhythms of eating and sleeping, expecting the provision of this kind of structure to facilitate the natural manifestation of satiety, rhythm, structure and regularity may be unrealistic. The parents who implement these types of structure and routine with seeming success may simply be exhibiting shared genetics with their child.

How much does parenting matter? Contrary to theories, as recently as the 1950's, we now know that 'bad parenting' does not cause autism or schizophrenia. Outside of abuse and neglect, usual adequate parenting may have modest impact on children's outcomes. As such, parenting may have relatively modest effects on childhood obesity risk. Until strong evidence from wellconducted randomized controlled trials shows a robust, reproducible effect of parenting on childhood obesity, the research community must be cautious about implying that childhood obesity is a result of suboptimal parenting-be it too permissive or not sensitive enough-usually by mothers.

\section{CONFLICT OF INTEREST}

The authors declare no conflict of interest. 


\section{ACKNOWLEDGEMENTS}

Dr Lumeng is supported by 5R01HD084163 from the National Institutes of Health.

JC Lumeng

Department of Pediatrics, University of Michigan-, Ann Arbor, MI, USA E-mail: jlumeng@umich.edu

\section{REFERENCES}

1 Anderson SE, Sacker A, Whitaker RC, Kelly Y. Self-regulation and household routines at ge three and obesity at age eleven: Longitudinal analysis of the UK Millennium cohort study. International J of Obesity; accepted article preview 24 April 2017; doi:10.1038/ijo.2017.94.

2 St-Onge M-P, Ard J, Baskin ML, Chiuve SE, Johnson HM, Kris-Etherton P et al. Meal timing and frequency: implications for cardiovascular disease prevention: a scientific statement from the American Heart Association. Circulation 2017; 135: e96-e121.

3 Sytsma SE, Kelley ML, Wymer JH. Development and initial validation of the child routines inventory. J Psychopathol Behav Assess 2001; 23: 241-251.
4 Lumeng JC, Miller AL, Horodynski MA, Brophy-Herb HE, Contreras D, Lee $\mathrm{H}$ et al. Improving self-regulation for obesity prevention in head start: a randomized controlled trial. Pediatrics 2017; 139: e20162047.

5 Loveman E, Al-Khudairy L, Johnson RE, Robertson W, Colquitt JL, Mead EL et al. Parent-only interventions for childhood overweight or obesity in children aged 5 to 11 years. Cochrane Database of Syst Rev 2015; (12): CD012008.

6 Sprunger LW, Boyce WT, Gaines JA. Family-infant congruence: routines and rhythmicity in family adaptations to a young infant. Child Dev 1985; 56: 564-572.

7 Wilson KE, Lumeng JC, Kaciroti N, SY-P Chen, LeBourgeois MK, Chervin RD et al. Sleep hygiene practices and bedtime resistance in low-income preschoolers: does temperament matter? Behav Sleep Med 2015; 13: 412-423.

8 Miller AL, Song JH, Sturza J, Lumeng JC, Rosenblum K, Kaciroti N et al. Child cortisol moderates the association between family routines and emotion regulation in low-income children. Dev Psychobiol 2017; 59: 99-110.

9 Fiese BH, Tomcho TJ, Douglas M, Josephs K, Poltrock S, Baker T. A review of 50 years of research on naturally occurring family routines and rituals: cause for celebration? J Fam Psychol 2002; 16: 381.

10 Rhee KE, Lumeng JC, Appugliese DP, Kaciroti N, Bradley RH. Parenting styles and overweight status in first grade. Pediatrics 2006; 117: 2047-2054.

11 Forehand R, McKinney B. Historical overview of child discipline in the United States: implications for mental health clinicians and researchers. J Child Fam Stud 1993; 2: 221-228. 